\title{
An unusual cause of lower intestinal bleeding-Leptospirosis
}

\author{
Sangar P, MBBS, MS ${ }^{1}$, Sagap I, MBBS, MS. ${ }^{2}$ \\ 1 Surgeon and Colorectal Fellow, Hospital Selayang, Malaysia. \\ 2 Professor and Consultant Colorectal Surgeon, National University of Malaysia, Kuala Lumpur, Malaysia.
}

Key words: Leptospirosis; Lower intestinal

bleed; Vasculitis; Anastomotic leak.

\begin{abstract}
A 37-year-old man was admitted with sepsis secondary to leptospirosis which was complicated with multiorgan failure, confirmed by a leptospira IgM positive test. He was treated with 2 weeks of meropenem and doxycycline. Two weeks later, he developed hematochezia. At colonoscopy, multiple haemorrhagic ulcers were noted in the terminal ileum. Despite intensive therapy, the bleeding worsened and laparascopic limited right hemicolectomy was done. An anastomotic leak occured secondary to severe vasculitis and, on day 3 , he underwent a defunctioning ileostomy and mucocutaneous fistula. Multiple ulcerated lesions were identified pathologically as vasculitis caused by leptospirosis. This is the second reported case of massive lower intestinal bleeding caused by leptospirosis[11].
\end{abstract}

\section{Introduction}

Lower gastrointestinal (GIT) haemorrhage refers to a spectrum of intestinal bleeding that arises distal to the ligament of Treitz. It may range from occult bleeding or occasional spotting of blood to massive lower GIT haemorrhage. Most often the massive lower GIT bleed is intermittent and may be due to a variety of causes. Lower GIT bleeding is always challenging for the surgeon in both diagnosis and in management.

Correspondence: Sangar Perumal, Surgeon and Colorectal Fellow, Hospital Selayang, Malaysia

Email: ptsangar@gmail.com

The Sri Lanka Journal of Surgery 2011; 29(1):34-36.
Leptospirosis is a widespread zoonosis with global distribution that is caused by pathogenic spirochete species of the genus Leptospira. The infection may be transmitted to humans by direct contact with infected tissues or contaminated urine, or indirectly by contact with water or moist soil contaminated with the urine of mammalian reservoirs. Severe manifestations occur in $510 \%$ of human infections and present mainly as Weil's syndrome, a triad of jaundice, hemorrhagic diathesis and acute renal failure (with 1015\% case fatality in most series) and severe pulmonary hemorrhage syndrome (SPHS), which may present as acute respiratory distress syndrome (ARDS) or massive pulmonary hemorrhage, with case fatality $>50 \%[1]$. Even though bleeding is a common feature of leptospirosis, massive lower GIT bleeding is uncommon. Besides bleeding, leptospirosis infection also causes focal or disseminated vasculitis and perivasculitis which may involve the lungs, heart, liver, spleen, and central nervous system $[2,3,4]$.

\section{Case report}

A 37-year-old male lorry driver with no previous medical illness was admitted with high grade fever and multiorgan failure (lungs and kidney) to a medical ward. He was diagnosed as having leptospirosis infection based on a positive leptospiral IgM antibody levels in serum. He was treated with meropenem and doxycycline and ventilated in intensive care unit for almost a week. Renal failure was treated by haemodialysis. After two weeks of hospitalization he developed massive hematochezia. He was referred to the 
colorectal unit where colonoscopy revealed multiple haemorrhagic ulcers in the terminal ileum.

Angio-embolisation was planned but the patient rebled and became hypotensive. An emergency laparascopic limited right hemicolectomy with an extracorporeal hand sewn anastomosis with vicryl $3 / 0$ was performed. Both edges were healthy, bleeding well and a post anastomosis leak test was negative. The opened specimen show edextensive mucosal ulceration of the terminal ileum. He was stable and well until day 3 post operation, where he developed fever, abdominal pain, tachycardia and peritonitis. Laparotomy and ileostomy with muco-cutaneous fistula was created because the entire anastomosishad given away; the anastomotic edges were unhealthy and had sloughed. The patient recovered well and was discharged on day 5 after laparotomy. He is currently on regular haemodialysis. The histopathology report of the resected specimen reported multifocal flaskshaped ulcers with vasculitis with dense infiltration of lymphocytes within the vessel walls in the submucosal layer. The histopathology of the refashioned edges of anastomosis also reported a severe vasculitis with necrosis.

\section{Discussion}

There are fourteen serotypes of leptospira. This infection is common in temperate and humid countries like Southeast Asia, the former Soviet Union, Japan, Nicaragua and Australia. After an incubation period of 10-21 days, the disease is characterized by fever, headache, myalgia, eschar with skin rash, and gastrointestinal symptoms. The reported severe complications of leptospirosis include meningoencephalitis [5], pneumonitis and acute respiratory distress syndrome [6], hepatitis [7], acute renal failure [8], upper gastrointestinal bleeding $[9,10]$ and necrotising pancreatitis.

Upper gastrointestinal bleeding associated with leptospirosis is reported in $10.3 \%$ of cases and, in $3.4 \%$, active bleeding that requires treatment with endoscopic clipping is reported [9]. In contrast, lower gastrointestinal bleeding associated with leptospirosis is rare with pathologically proven vasculitis [11].

This lower GIT bleeding which was caused by leptospirosis is commonly assumed to cause systemic vasculitis and perivasculitis which would be one of the main mechanisms of tissue damage. Inflammation of the vascular walls may be a consequence of the direct invasion of infectious agents, or of immune mechanisms such as immune complex deposition, auto-antibodies and cell-mediated immunity. There is also the presence of endothelial lesions found by ultrastructure study of leptospirosis infected blood vessels [12]. Besides diffuse endothelial damage and systemic inflammation, reports of true attacks on arterial walls are also available. Marked inflammation in the aortic adventitia was noted in $4 / 33(12 \%)$ and $11 / 19(58 \%)$, and coronary inflammation in $2 / 33(6 \%)$ and $14 / 20$ $(70 \%)$ patients, in human necropsy series [12]. There are some descriptions of the pathologic anatomy of cerebral arteritis in leptospirosis from China [13,14]. The inflammation in this disease may involve all layers of the main branches of large arteries in the base of the brain, and it was found to occur in both children and adolescents, leading to a multiple occlusive

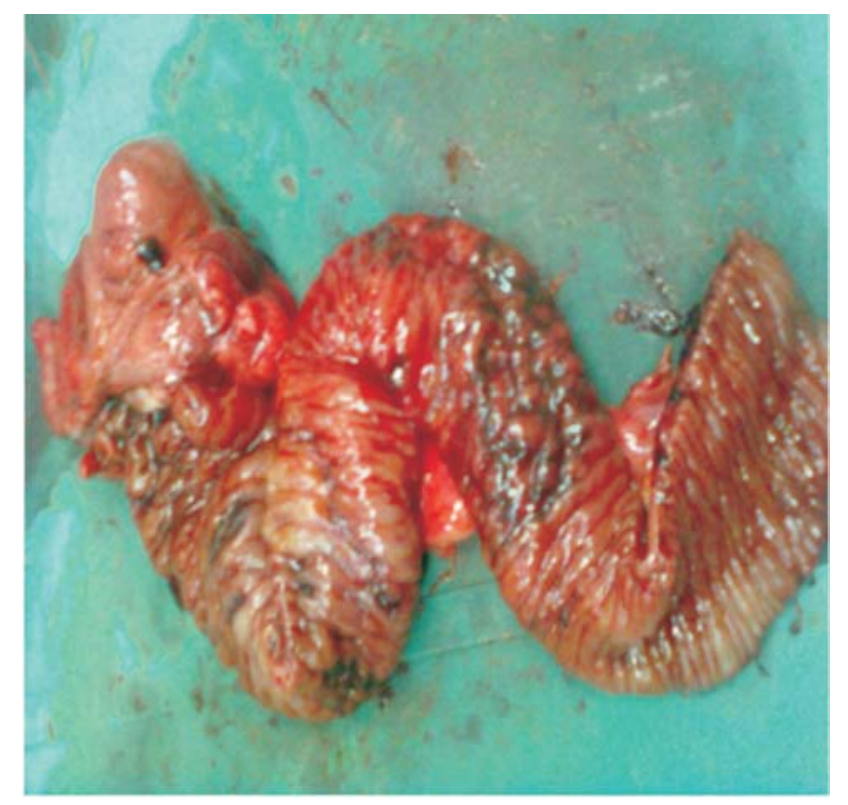

Figure 1. The terminal ileum; black patches are bleeding ulcers. 
cerebrovascular disease in $75 \%$ of cases in the largest series of cerebrovascular leptospirosis [13].

All of this evidence can explain causes of bleeding in leptospirosis. Besides that, one should be cautious of primary anastomosis in active disease where, there is severe vasculitis as well as endothelial damage of vessels. This explains the anastomotic leak in this patient. More extensivestudy of blood vessel and systemic response in leptospirosis is needed to further understand the disease.

\section{References}

1. McBride AJ, Athanazio DA, Reis MG and Ko AI, Leptospirosis, Curr. Opin. Infect. Dis. 2005;18:376-386.

2. Saah AJ. Orientia tsutsugamushi(scrub typhus). In: Mandell GL, Bennett JE, Dolin $\mathrm{R}$, editors. Principles and practice of infectious disease. 5th ed. Philadelphia, Pa: Churchill Livingstone, 2000: 2056-2057.

3. Settle EB, Pinkerton H, Corbett AJ. A pathologic study of tsutsugamushi disease (scrub typhus) with notes on clinicopathologic correlation. J Lab Clin Med 1945; 30:639-661.

4. Levine HD. Pathologic study of thirty-one cases of scrub typhus fever with especial reference to the cardiovascular system. Am Heart J 1945; 31:314-328.

5. Silpapojakul K, Ukkachoke C, Krisanapan S, Silpapojakul K. Rickettsial meningitis and encephalitis. Arch Intern Med 1991; 151:1753-1757.

6. Walker DH, Mattern WD. Rickettsial vasculitis. Am Heart J 1980; 100:896-906.
7. Chien RN, Liu NJ, Lin PY, Liaw YF. Granulomatous hepatitis associated with scrub typhus. J Gastroenterol Hepatol 1995; 10:484-487.

8. Yen TH, Chang CT, Lin JL, Jiang JR, Lee KF. Scrub typhus: a frequently overlooked cause of acute renal failure. Ren Fail 2003; 25:397-410.

9. Aung-Thu, Supanaranond W, Phumiratanaprapin W, Phonrat B, Chinprasatsak S, Ratanajaratroj N. Gastrointestinal manifestations of septic patients with scrub typhus in Maharat Nakhon Ratchasima Hospital. Southeast Asian J Trop Med Public Health 2004; 35:845-851.

10. Kim SJ, Chung IK, Chung IS, Song DH, Park SH, Kim HS, Lee MH. The clinical significance of upper gastrointestinal endoscopy in gastrointestinal vasculitis related to scrub typhus. Endoscopy 2000; 32:950-955.

11. Ki Beom Bae, Won Hwa Youn, Youn Jae Lee, Soo Jin Jung, Kwan Hee Hong. Massive small bowel bleeding caused by scrub typhus in Korea.World J Gastrointest Surg 2010; 2(2):47-50.

12. T. De Brito, G.M. Bohm and P.H. Yasuda, Vascular damage in acute experimental leptospirosis of the guinea-pig, J. Pathol. $1979 ; 128: 177-182$ Y.

13. Chen, A clinicopathological analysis of 12 cases of cerebrovascular leptospirosis, Chung Hua Shen Ching Ching Shen Ko Tsa Chih 1990; 23:226-228.

14. Gong Y.H, Leptospiral cerebral arteritis: report of 4 autopsy cases, Acta Acad. Med. Wuhan 1984; 4:44-49.

\section{Key Learning Points}

Leptospirosis may cause massive lower intestinal bleeding secondary to vasculitis.

A lower intestinal bleed may be treated with a laparascopic approach.

Be cautious of primary bowel anastomosis in the presence of vasculitis. 